\title{
ON THE GROWTH ORDER OF AN ALGEBROID FUNCTION WITH RADIALLY DISTRIBUTED VALUES IN THE UNIT DISK
}

\author{
NAN WU AND JIAN-HUA ZHENG
}

(Received March 28, 2013, revised August 9, 2013)

\begin{abstract}
For an algebroid function in the unit disk of finite lower order with a deficient value, we can estimate its growth order in terms of the convergence exponent of the points of the deficient value and other distinct values not lying on a radial system and the maximal difference of the arguments of adjacent rays.
\end{abstract}

1. Introduction and main results. We assume that the reader is familiar with the fundamental results and standard notations of the Nevanlinna theory in the unit disk $\Delta=$ $\{z ;|z|<1\}$ and in the complex plane $C$ (see [4, 9, 13, 17]). A value $a$ on the extended complex plane $\widehat{\boldsymbol{C}}=\boldsymbol{C} \cup\{\infty\}$ is called a radially distributed value of a transcendental meromorphic function if most of points at which the value is assumed distribute closely along a finite number of rays from the origin. Many people have taken into account how the growth of a meromorphic function could be affected by distribution of the arguments of its value $a$-points in the complex plane.

In 1955, Edrei [3] proved the following theorem.

THEOREM A ([3]). Let $f(z)$ be a meromorphic function in $\boldsymbol{C}$ and such that the roots of the three equations

$$
f(z)=0, \quad f(z)=\infty, \quad f^{(l)}(z)=1 \quad\left(l \geq 0, f^{(0)} \equiv f\right)
$$

are distributed on the rays

$$
r e^{i \omega_{1}}, r e^{i \omega_{2}}, \ldots, r e^{i \omega_{q}} \quad\left(r \geq 0,0 \leq \omega_{1}<\omega_{2}<\cdots<\omega_{q}<2 \pi\right) .
$$

Denote by $\delta\left(a, f^{(l)}\right)$ the deficiency of the value a of the function $f^{(l)}$, and assume $\delta(0, f)+$ $\delta\left(1, f^{(l)}\right)+\delta(\infty, f)>0$. Then the order $\lambda(f)$ of $f(z)$ is necessarily finite and

$$
\lambda(f) \leq \beta=\sup _{1 \leq i \leq q}\left\{\pi /\left(\omega_{i+1}-\omega_{i}\right)\right\} \quad\left(\omega_{q+1}=2 \pi+\omega_{1}\right) .
$$

In 1993, Wu [10] used the Nevanlinna theory of meromorphic functions in angular domains to study this problem and obtained the following theorem.

2000 Mathematics Subject Classification. Primary 30D10; Secondary 30D20, 30B10, 34M05.

Key words and phrases. Algebroid function, order, deficient value. 
THEOREM B ([10]). Let $f(z)$ be a meromorphic function of finite lower order $\mu$ in C. Suppose that $\arg z=\theta_{j}\left(j=1,2, \ldots, q ; 1 \leq q<\infty ; 0 \leq \theta_{1}<\theta_{2}<\cdots<\theta_{q}<\right.$ $\left.2 \pi, \theta_{q+1}=\theta_{1}+2 \pi\right)$ are $q$ rays in $\boldsymbol{C}$, such that for any $\varepsilon>0$ and $x=0, \infty$, we have

$$
\limsup _{r \rightarrow \infty} \frac{\log n\left(r, \cup_{j=1}^{q} \Omega\left(\theta_{j}+\varepsilon, \theta_{j+1}-\varepsilon\right), f=x\right)}{\log r} \leq \rho<\infty,
$$

where $\rho$ is a positive number, $\Omega\left(\theta_{j}+\varepsilon, \theta_{j+1}-\varepsilon\right)=\left\{z ; \theta_{j}+\varepsilon<\arg z<\theta_{j+1}-\varepsilon\right\}$, $n(r, X, f=x)$ denotes the number of the roots of $f(z)=x$ in the region $X \cap\{|z| \leq r\}(X \subset$ $C)$, counting multiplicities. If $f^{(l)}$ has a finite and nonzero deficient value $a$, then the order of $f(z)$ satisfies

$$
\lambda(f) \leq \max \left(\frac{\pi}{\omega}, \rho\right),
$$

where $\omega=\min _{1 \leq j \leq q}\left(\theta_{j+1}-\theta_{j}\right)$.

In 2003, Zheng [19] gave a simple and elementary way to study the growth of transcendental meromorphic functions in terms of their orders when they and their derivatives have radially distributed values and proved the following theorem.

THEOREM C ([19]). Let $f(z)$ be a transcendental meromorphic function of finite lower order $\mu$ in $\boldsymbol{C}$ such that for some $a \in \widehat{\boldsymbol{C}}$ and an integer $p \geq 0, \delta=\delta\left(a, f^{(p)}\right)>0$. If for q pair $\left\{\alpha_{j}, \beta_{j}\right\}$ of real numbers satisfying

$$
-\pi \leq \alpha_{1}<\beta_{1}<\alpha_{2}<\beta_{2}<\cdots<\alpha_{q}<\beta_{q} \leq \pi
$$

and an integer $k>0$, we have

$$
\limsup _{r \rightarrow \infty} \frac{\log \left[n(r, Y, f=0)+n\left(r, Y, f^{(k)}=1\right)\right]}{\log r} \leq \rho,
$$

for $Y=\bigcup_{j=1}^{q}\left\{z ; \alpha_{j} \leq \arg z \leq \beta_{j}\right\}$ and

$$
\sum_{j=1}^{q}\left(\alpha_{j+1}-\beta_{j}\right)<\frac{4}{\beta} \arcsin \sqrt{\frac{\delta}{2}}, \quad \alpha_{q+1}=\alpha_{1}+2 \pi,
$$

where $\beta=\max \{\rho, \omega, \mu\}, \omega=\max _{1 \leq j \leq q}\left\{\pi /\left(\beta_{j}-\alpha_{j}\right)\right\}$, then $\lambda(f) \leq \max \{\omega, \rho\}$.

In 2006, Chang [1] used another method to study the radially distributed values of meromorphic functions and obtained the following theorem.

THEOREM D ([1]). Let $f(z)$ be a meromorphic function in $C$ of finite lower order with $\delta(0, f)+\delta(\infty, f)>0$. Suppose that f has no direction of Marty-type except $L: \arg z=$ $\theta_{j}\left(j=1,2, \ldots, q ; 0 \leq \theta_{1}<\theta_{2}<\cdots<\theta_{q}<2 \pi\right)$. Then the order of $f$ satisfies

$$
\lambda(f) \leq \frac{\pi}{\omega},
$$

where $\omega=\min _{1 \leq j \leq q}\left(\theta_{j+1}-\theta_{j}\right)$.

The definition of a Marty direction of a meromorphic function can be seen in [1]. In [18], Zheng suggested that there are three aspects for an algebroid function worthy of consideration, 
one of them being the growth of an algebroid function when some restriction is imposed on arguments of certain $a$-points. We considered this problem for an algebroid function $f(z)$ defined in the complex plane in another article [12]. In this note, we will investigate the growth of an algebroid function with radially distributed values in the unit disk. Before stating the result, we give some notations and definitions of an algebroid function.

Let $f=f(z)(z \in \Delta)$ be the $v$-valued algebroid function defined by an irreducible equation

$$
F(z, w):=A_{0}(z) w^{\nu}+A_{1}(z) w^{\nu-1}+\cdots+A_{v}(z)=0,
$$

where $A_{v}(z), \ldots, A_{0}(z)$ are analytic functions without any common zeros in the unit disk. Let $\vec{A}=\left(A_{0}, \ldots, A_{v}\right), \vec{\infty}=(1,0, \ldots, 0)$. For any $a \in C$, denote $\vec{a}=\left(a^{v}, a^{\nu-1}, \ldots, 1\right)$. Then,

$$
\begin{gathered}
\|\vec{A}(z)\|=\left(\left|A_{0}\right|^{2}+\left|A_{1}\right|^{2}+\cdots+\left|A_{\nu}\right|^{2}\right)^{1 / 2}, \\
\|\vec{a}\|= \begin{cases}\left(|a|^{2 v}+|a|^{2 v-2}+\cdots+|a|^{2}+1\right)^{1 / 2}, & a \neq \infty, \\
1, & a=\infty .\end{cases}
\end{gathered}
$$

Since $F(z, w)$ is irreducible, $F(z, a)=\vec{A}(z) \cdot \vec{a} \not \equiv 0$, where $F(z, \infty)=A_{0}(z)$. Set

$$
\log ^{+} x=\max \{0, \log x\} .
$$

Define the proximity function to a number $a \in \widehat{\boldsymbol{C}}$ of $f(z)$ on the circle $\{|z|=r\}$ :

$$
m(r, \vec{a}, \vec{A})=\frac{1}{2 \pi} \int_{0}^{2 \pi} \log ^{+} \frac{\left\|\vec{A}\left(r e^{i \theta}\right)\right\|\|\vec{a}\|}{\left|F\left(r e^{i \theta}, a\right)\right|} d \theta,
$$

and the counting function of $a$-points:

$$
\begin{aligned}
N(r, \vec{a}, \vec{A}) & =N(r, 0, F(z, a)) \\
& =\int_{0}^{r} \frac{n(t, 0, F(z, a))-n(0,0, F(z, a))}{t} d t+n(0,0, F(z, a)) \log r,
\end{aligned}
$$

where $n(t, 0, F(z, a))$ is the number of the roots of the equation $F(z, a)=0$ in the disk $\{|z| \leq t\}$, counting multiplicities. In this paper, $n(t, a, f(z))$ denotes the number of the roots of $f(z)=a$ in $\{|z| \leq t\}$, counting multiplicities. Put

$$
T(r, \vec{a}, \vec{A})=m(r, \vec{a}, \vec{A})+N(r, \vec{a}, \vec{A}) .
$$

Following G.Valiron, we define the characteristic function of $f(z)$ as

$$
T(r, f)=\frac{1}{2 \nu \pi} \int_{0}^{2 \pi} \log \max _{0 \leq j \leq \nu}\left|A_{j}\left(r e^{i \theta}\right)\right| d \theta .
$$

By using Valiron's result (cf. [15]), we get the relation between $T(r, f)$ and $T(r, \vec{a}, \vec{A})$ :

$$
|T(r, \vec{a}, \vec{A})-v T(r, f)|=O(1) .
$$

The counting function of $a$-points of $f(z)$ is defined as

$$
N(r, a, f)=\frac{1}{v} N(r, 0, F(z, a)) .
$$


Put

$$
\delta(a, f)=1-\limsup _{r \rightarrow 1-} \frac{N(r, a, f)}{T(r, f)}=1-\limsup _{r \rightarrow 1-} \frac{N(r, 0, F(z, a))}{T(r, \vec{a}, \vec{A})} .
$$

The value $a$ is called a Nevanlinna deficient value of $f$ if $\delta(a, f)>0$. The order and lower order of $f(z)$ are defined as

$$
\lambda(f):=\limsup _{r \rightarrow 1-} \frac{\log ^{+} T(r, f)}{\log (1-r)^{-1}} ; \quad \mu(f):=\liminf _{r \rightarrow 1-} \frac{\log ^{+} T(r, f)}{\log (1-r)^{-1}} .
$$

Given a sector $Y=\{z ; \alpha<\arg z<\beta,|z|<1\}$. Define the counting function of $a$-points of $f(z)$ in $Y$ as

$$
N(r, Y, f=a)=\frac{1}{v} \int_{0}^{r} \frac{n(t, Y, f=a)}{t} d t,
$$

where $n(t, Y, f=a)$ is the number of the roots of $f(z)=a$ in $Y \cap\{z ;|z|<t\}$, counting multiplicities. Now we can state our result as follows.

THEOREM 1.1. Let $f(z)$ be the $v$-valued algebroid function of finite lower order $\mu$ in $\Delta$ determined by $(1.1)$ and such that $\delta(a, f)>0$ for some $a \in \widehat{\boldsymbol{C}}$. Suppose that

$$
\arg z=\theta_{k} \quad\left(k=1,2, \ldots, q, 0 \leq \theta_{1}<\theta_{2}<\cdots<\theta_{q}<\theta_{1}+2 \pi=\theta_{q+1}\right)
$$

are $q(1 \leq q<+\infty)$ radii in the unit disk, and there exist $2 v$ distinct values $a_{i} \neq a(i=$ $1,2, \ldots, 2 v)$ such that

$$
\limsup _{r \rightarrow 1-} \frac{\log \left[\sum_{i=1}^{2 v} n\left(r, Y, f=a_{i}\right)+n(r, Y, f=a)\right]}{\log (1-r)^{-1}} \leq \rho<\infty,
$$

where $Y=\Delta \backslash \bigcup_{k=1}^{q}\left\{z ; \arg z=\theta_{k},|z|<1\right\}$ and $\rho>1$. Then, the order of $f$ satisfies

$$
\lambda(f) \leq \rho+2+\max _{1 \leq k \leq q}\left(\frac{\theta_{k+1}-\theta_{k}}{\pi}\right) .
$$

We complete the proof of Theorem 1.1 with the help of the method in Yang [14] and Chang [1] and the behavior of algebroid functions in the unit disk. Both the papers investigate the growth of transcendental meromorphic functions with radially distributed values in the complex plane.

2. Some lemmas. In this section we give some lemmas which are used in the proofs of our theorems.

LEMMA 2.1 ([11]). Let $\varphi(r)$ be a nondecreasing and positive real function in $(0,1)$ and satisfy

$$
\limsup _{r \rightarrow 1-} \frac{\varphi(r)}{\log (1-r)^{-1}}=\varrho \leq+\infty .
$$

Then, for any set $E \subset(0,1)$ such that $\int_{E} \frac{d r}{1-r}<\infty$, we have

$$
\limsup _{r \rightarrow 1-, r \in(0,1) \backslash E} \frac{\varphi(r)}{\log (1-r)^{-1}}=\varrho \leq+\infty .
$$


The proof of Lemma 2.1 can be seen in [11].

\section{LEMMA 2.2. The transformation}

$$
u(z)=\frac{\left(z e^{-i \theta_{0}}\right)^{\pi / \delta}+2\left(z e^{-i \theta_{0}}\right)^{\pi /(2 \delta)}-1}{\left(z e^{-i \theta_{0}}\right)^{\pi / \delta}-2\left(z e^{-i \theta_{0}}\right)^{\pi /(2 \delta)}-1}, \quad\left(0 \leq \theta_{0}<2 \pi, 0<\delta<\pi\right)
$$

is a conformal map of the sector $\Omega\left(\theta_{0}-\delta, \theta_{0}+\delta\right)=\left\{z ; \theta_{0}-\delta<\arg z<\theta_{0}+\delta,|z|<1\right\}$ onto the unit disc $\Delta$. For any positive number $\varepsilon$ satisfying $0<\varepsilon<\delta$, the transformation (2.1) satisfies

$$
\begin{aligned}
& u\left(\left\{z ; 2^{-1}<|z|<r\right\} \cap\left\{z ;\left|\arg z-\theta_{0}\right|<\delta-\varepsilon\right\}\right) \subset\left\{u ;|u|<1-\frac{\varepsilon}{2^{\frac{\pi}{2 \delta}}+1}(1-r)\right\} ; \\
& u^{-1}(\{u ;|u|<\rho\}) \subset\left(\left\{z ;|z|<1-\frac{\delta}{8 \pi}(1-\rho)\right\} \cap\left\{z ;\left|\arg z-\theta_{0}\right|<\delta\right\}\right) .
\end{aligned}
$$

The inverse transformation of (2.1) is

$$
z=e^{i \theta_{0}}\left[\frac{-(1+u)+\sqrt{2\left(1+u^{2}\right)}}{1-u}\right]^{2 \delta / \pi}
$$

If we regard (2.3) as a map from the rectangle $[0,1) \times[0,2 \pi)$ on $|u|-\arg u$ plane onto the rectangle $(0,1) \times\left(\theta_{0}-\delta, \theta_{0}+\delta\right)$ on $|z|-\arg z$ plane, then the Jacobian satisfies

$$
|J|=O\left(\frac{1}{(1-|u|)^{2 \delta / \pi+3}}\right), \quad|u| \rightarrow 1-.
$$

In fact, the proof of (2.2) has been given by Sun [7] and Zhang [16] respectively. However, they only prove the case of $\varepsilon=\delta / 2$. Here, we will prove (2.2) for the case of $0<\varepsilon<\delta$ by the same method of Zhang [16]. Moreover, we give the proof of (2.4) for the first time.

Proof. Set $z=|z| e^{i \varphi}, \alpha=\pi /(2 \delta)>1$. From (2.1), we have

$$
1-|u|^{2}=\frac{8\left(1-|z|^{2 \alpha}\right)|z|^{\alpha} \cos \alpha\left(\varphi-\theta_{0}\right)}{\left|\left(z e^{-\theta_{0}}\right)^{2 \alpha}-2\left(z e^{-\theta_{0}}\right)^{\alpha}-1\right|^{2}} .
$$

When $2^{-1}<|z|<r$ and $\left|\arg z-\theta_{0}\right|<\delta-\varepsilon$,

$2(1-|u|)>1-|u|^{2} \geq \frac{8(1-|z|)\left(\frac{1}{2}\right)^{\alpha} \sin (\alpha \varepsilon)}{(1+2+1)^{2}}>\frac{8(1-|z|)\left(\frac{1}{2}\right)^{\alpha} \frac{2}{\pi}(\alpha \varepsilon)}{(1+2+1)^{2}}=\frac{\varepsilon}{2^{\alpha+1} \delta}(1-|z|)$.

Then, $|u|<1-\frac{\varepsilon}{2^{\alpha+1} \delta}(1-|z|)<1-\frac{\varepsilon}{2^{\alpha+1} \delta}(1-r)$.

After a simple calculation, we find that when $|u|<\rho$,

$$
1-\rho<1-|u|^{2}<16 \alpha(1-|z|) .
$$

Then, $|z|<1-\frac{\delta}{8 \pi}(1-\rho)$. Thus $(2.2)$ is proved.

Next we will prove (2.4). Write $z=r e^{i \theta}, u=t e^{i \phi}$. Taking the derivative on both side of (2.3), we get that

$$
\frac{d z}{d u}=\frac{e^{i \theta_{0}}}{\alpha}\left[\frac{-(1+u)+\sqrt{2\left(1+u^{2}\right)}}{1-u}\right]^{1 / \alpha-1} \frac{\sqrt{2}(u+1)-2 \sqrt{1+u^{2}}}{(1-u)^{2} \sqrt{1+u^{2}}} .
$$


Taking the partial derivatives on both side of (2.3), we derive

$$
\begin{aligned}
\frac{\partial r}{\partial t} e^{i \theta} & +i \frac{\partial \theta}{\partial t} r e^{i \theta} \\
& =\frac{e^{i \theta_{0}}}{\alpha}\left[\frac{-(1+u)+\sqrt{2\left(1+u^{2}\right)}}{1-u}\right]^{1 / \alpha-1} \frac{\sqrt{2}(u+1)-2 \sqrt{1+u^{2}}}{(1-u)^{2} \sqrt{1+u^{2}}} \cdot e^{i \phi},
\end{aligned}
$$

$$
\begin{aligned}
\frac{\partial r}{\partial \phi} e^{i \theta} & +i \frac{\partial \theta}{\partial \phi} r e^{i \theta} \\
= & \frac{e^{i \theta_{0}}}{\alpha}\left[\frac{-(1+u)+\sqrt{2\left(1+u^{2}\right)}}{1-u}\right]^{1 / \alpha-1} \frac{\sqrt{2}(u+1)-2 \sqrt{1+u^{2}}}{(1-u)^{2} \sqrt{1+u^{2}}} \cdot t i e^{i \phi}
\end{aligned}
$$

Combining (2.6) with (2.7) gives

$$
\frac{\frac{\partial r}{\partial t}+i \frac{\partial \theta}{\partial t} r}{\frac{\partial r}{\partial \phi}+i \frac{\partial \theta}{\partial \phi} r}=\frac{1}{i t}
$$

so that

$$
|J|=\left|\frac{\partial r}{\partial t} \frac{\partial \theta}{\partial \phi}-\frac{\partial r}{\partial \phi} \frac{\partial \theta}{\partial t}\right|=\frac{\left|\frac{\partial r}{\partial \phi} e^{i \theta}+i \frac{\partial \theta}{\partial \phi} r e^{i \theta}\right|^{2}}{t r} .
$$

Noticing that

$$
r=|z|=\left|\frac{-(1+u)+\sqrt{2\left(1+u^{2}\right)}}{1-u}\right|^{1 / \alpha}
$$

then from (2.7) it follows that

$$
\frac{\left|\frac{\partial r}{\partial \phi} e^{i \theta}+i \frac{\partial \theta}{\partial \phi} r e^{i \theta}\right|^{2}}{r}=\frac{1}{\alpha^{2}}\left|\frac{-(1+u)+\sqrt{2\left(1+u^{2}\right)}}{1-u}\right|^{1 / \alpha-2}\left|\frac{\sqrt{2}(u+1)-2 \sqrt{1+u^{2}}}{(1-u)^{2} \sqrt{1+u^{2}}}\right|^{2} t^{2} .
$$

Therefore,

$$
\begin{aligned}
|J| & =\frac{\left|\frac{\partial r}{\partial \phi} e^{i \theta}+i \frac{\partial \theta}{\partial \phi} r e^{i \theta}\right|^{2}}{r t} \\
& =\frac{t}{\alpha^{2}}\left|\frac{-(1+u)+\sqrt{2\left(1+u^{2}\right)}}{1-u}\right|^{1 / \alpha-2}\left|\frac{\sqrt{2}(u+1)-2 \sqrt{1+u^{2}}}{(1-u)^{2} \sqrt{1+u^{2}}}\right|^{2} \\
& \leq \frac{t}{\alpha^{2}}\left|-(1+u)+\sqrt{2\left(1+u^{2}\right)}\right|^{1 / \alpha-2}\left|\sqrt{2}(u+1)-2 \sqrt{1+u^{2}}\right|^{2} \frac{1}{(1-|u|)^{1 / \alpha+3}} \\
& \leq \frac{4^{1 / \alpha-2} \cdot(2 \sqrt{2}+2 \sqrt{2})^{2}}{\alpha^{2}} \frac{1}{(1-|u|)^{1 / \alpha+3}} .
\end{aligned}
$$

Thus, as $|u|=t \rightarrow 1-$, we have

$$
|J| \leq \frac{2^{2 / \alpha+1}}{\alpha^{2}} \frac{1}{(1-t)^{1 / \alpha+3}} .
$$

Hence the proof of Lemma 2.2 is completed. 
The following lemma is the second fundamental theorem for algebroid functions in the unit disk, whose proof can be found in $[6,8]$, and we can obtain the error term $S(r, f)$ by the same method as used in meromorphic functions.

LEMMA 2.3 ([6,8]). Let $f(z)$ be the v-valued algebroid function in $\Delta$ determined by (1.1) and let $a_{1}, a_{2}, \ldots, a_{q}$ be $q$ different values on $\widehat{\boldsymbol{C}}$. Then, we have

$$
(q-2 v) T(r, f)<\sum_{i=1}^{q} N\left(r, a_{i}, f\right)+S(r, f),
$$

where

$$
S(r, f)= \begin{cases}O\left(\log (1-r)^{-1}\right) & \text { if } \lambda(f)<\infty, \\ O\left(\log (1-r)^{-1}+\log T(r, f)\right), r \notin F & \text { if } \lambda(f)=\infty,\end{cases}
$$

where $F$ is a set satisfying that $F \subset(0,1)$ and $\int_{F} d r /(1-r)<\infty$.

LEMMA 2.4. Let $f(z)$ be the v-valued algebroid function in $\Delta$ determined by (1.1). Suppose that there exist $2 v+1$ distinct complex values $a_{i}(i=1,2, \ldots, 2 v)$, a such that for any small $\varepsilon>0$, we have

$$
\limsup _{r \rightarrow 1-} \frac{\log \left[\sum_{j=1}^{2 v} n\left(r, Y, f=a_{j}\right)+n(r, Y, f=a)\right]}{\log (1-r)^{-1}} \leq \rho+1<\infty,
$$

where $Y=\left\{z ; \theta_{1}+\varepsilon<\arg z<\theta_{2}-\varepsilon,|z|<1\right\}$ and $\rho>0$. Then

$$
\frac{1}{2 \pi} \int_{\theta_{1}+2 \varepsilon}^{\theta_{2}-2 \varepsilon} \log +\frac{\left\|\vec{A}\left(r e^{i \theta}\right)\right\|\|\vec{a}\|}{\left|F\left(r e^{i \theta}, a\right)\right|} d \theta \leq\left(\frac{1}{1-r}\right)^{\rho+1 / \omega+3+2 \varepsilon} \log ^{2}\left(\frac{1}{1-r}\right)
$$

holds except a set $E \subset(0,1)$ satisfying $\int_{E} d r /(1-r)<\infty$, where $\omega=\pi /\left(\theta_{2}-\theta_{1}-2 \varepsilon\right)$.

PROOF. By Lemma 2.2, we know that the transformation

$$
z=z(u)=e^{i \frac{\theta_{1}+\theta_{2}}{2}}\left[\frac{-(1+u)+\sqrt{2\left(1+u^{2}\right)}}{1-u}\right]^{\frac{\theta_{2}-\theta_{1}-2 \varepsilon}{\pi}}
$$

is a conformal map of the unit disk to the sector $Y$. Set $\omega=\frac{\pi}{\theta_{2}-\theta_{1}-2 \varepsilon}$. For $t<1$, we have

$$
z(\{u ;|u|<t\}) \subset\left\{z ; \theta_{1}+\varepsilon<\arg z<\theta_{2}-\varepsilon,|z|<1-\frac{1}{16 \omega}(1-t)\right\} .
$$

Since the number of roots of a equation in a region is a conformal invariant, it follows that

$$
\begin{aligned}
n(t, \tau, f(z(u))) & \leq n\left(1-\frac{1}{16 \omega}(1-t), Y, f(z)=\tau\right) \\
& =O\left(\frac{1}{(1-t)^{\rho+1+\varepsilon}}\right), \quad t \rightarrow 1-, \quad \tau=a_{j}(j=1,2, \ldots, 2 \nu), a .
\end{aligned}
$$


After a calculation, we obtain

$$
\begin{aligned}
N(t, \tau, f(z(u))) & =\frac{1}{v} \int_{0}^{t} \frac{n(x, \tau, f(z(u)))}{x} d x \\
& =\frac{1}{v} \int_{1 / 2}^{t} \frac{n(x, \tau, f(z(u)))}{x} d x+O(1) \\
& \leq \frac{2}{v} \int_{1 / 2}^{t} n(x, \tau, f(z(u))) d x+O(1) \\
& \leq O\left(\int_{1 / 2}^{t} \frac{1}{(1-x)^{\rho+1+\varepsilon}} d x\right)+O(1) \\
& =O\left(\frac{1}{(1-t)^{\rho+\varepsilon}}\right), \quad t \rightarrow 1-, \quad \tau=a_{j}(j=1,2, \ldots, 2 v), a .
\end{aligned}
$$

For $f(z(u))$ and complex number $a_{j}(j=1,2, \ldots, 2 v), a$ we apply Lemma 2.3,

$$
\begin{aligned}
T(t, f(z(u))) \leq & \sum_{i=1}^{2 v} N\left(t, a_{i}, f(z(u))\right)+N(t, a, f(z(u))) \\
& +O\left(\log (1-t)^{-1}+\log T(t, f(z(u)))\right) \\
\leq & O\left(\frac{1}{(1-t)^{\rho+\varepsilon}}\right)+O(\log T(t, f(z(u)))) \\
< & O\left(\frac{1}{(1-t)^{\rho+\varepsilon}}\right)+\frac{1}{2} T(t, f(z(u))), \quad t \notin F, \quad t \rightarrow 1-.
\end{aligned}
$$

So that

and

$$
T(t, f(z(u)))<O\left(\frac{1}{(1-t)^{\rho+\varepsilon}}\right), \quad t \notin F, \quad t \rightarrow 1-,
$$

$$
\limsup _{t \notin F, t \rightarrow 1-} \frac{\log T(t, f(z(u)))}{\log (1-t)^{-1}} \leq \rho<\infty .
$$

By Lemma 2.1, we have $\lambda(f(z(u))) \leq \rho<\infty$. Combining the first case of Lemma 2.3 and (2.9) gives

$$
\begin{aligned}
T(t, f(z(u))) & \leq \sum_{i=1}^{2 v} N\left(t, a_{i}, f(z(u))\right)+N(t, a, f(z(u)))+O\left(\log (1-t)^{-1}\right) \\
& \leq O\left(\frac{1}{(1-t)^{\rho+\varepsilon}}\right)+O\left(\log (1-t)^{-1}\right) \leq O\left(\frac{1}{(1-t)^{\rho+\varepsilon}}\right)
\end{aligned}
$$

Then,

$$
m(t, \vec{a}, \vec{A}(z(u))) \leq \nu T(t, f(z(u)))+O(1) \leq O\left(\frac{1}{(1-t)^{\rho+\varepsilon}}\right) .
$$

Using Lemma 2.2 for the transformation (2.8), we have $u\left(\left\{z ; 2^{-1}<|z|<r, \theta_{1}+2 \varepsilon<\arg z<\theta_{2}-2 \varepsilon\right\}\right) \subset\left\{u ;|u|<1-\frac{\varepsilon}{2^{\alpha}\left(\theta_{2}-\theta_{1}-2 \varepsilon\right)}(1-r)\right\}$, 
and the Jacobian determinant satisfies that

$$
|J| \leq O\left(\frac{1}{(1-r)^{1 / \omega+3}}\right) .
$$

Combining (2.11) with (2.12) it follows that

$$
\begin{aligned}
H & :=\int_{2^{-1}}^{r} \int_{\theta_{1}+2 \varepsilon}^{\theta_{2}-2 \varepsilon} \log +\frac{\left\|\vec{A}\left(r e^{i \theta}\right)\right\|\|\vec{a}\|}{\left|F\left(r e^{i \theta}, a\right)\right|} d \theta d r \\
& \leq \int_{0}^{1-\frac{\varepsilon}{2^{\alpha}\left(\theta_{2}-\theta_{1}-2 \varepsilon\right)}(1-r)} \int_{0}^{2 \pi} \log ^{+} \frac{\left\|\vec{A}\left(z\left(t e^{i \phi}\right)\right)\right\|\|\vec{a}\|}{\left|F\left(z\left(t e^{i \phi}\right), a\right)\right|} \frac{1}{(1-t)^{1 / \omega+3}} d \phi d t \\
& =\int_{0}^{1-\frac{\varepsilon}{2^{\alpha}\left(\theta_{2}-\theta_{1}-2 \varepsilon\right)}(1-r)} m(t, \vec{a}, \vec{A}(z(u))) \frac{d t}{(1-t)^{1 / \omega+3}} \\
& =\int_{0}^{1-\frac{\varepsilon}{2^{\alpha}\left(\theta_{2}-\theta_{1}-2 \varepsilon\right)}(1-r)}\left(\frac{1}{1-t}\right)^{\rho+1 / \omega+3+\varepsilon} d t=O\left(\left(\frac{1}{1-r}\right)^{\rho+1 / \omega+2+\varepsilon}\right) \\
& <\left(\frac{1}{1-r}\right)^{\rho+1 / \omega+2+2 \varepsilon} .
\end{aligned}
$$

Set

$$
E=\left\{0<r<1 ; \frac{1}{2 \pi} \int_{\theta_{1}+2 \varepsilon}^{\theta_{2}-2 \varepsilon} \log +\frac{\left\|\vec{A}\left(r e^{i \theta}\right)\right\|\|\vec{a}\|}{\left|F\left(r e^{i \theta}, a\right)\right|} d \theta>\left(\frac{1}{1-r}\right)^{\rho+1 / \omega+3+2 \varepsilon} \log ^{2}\left(\frac{1}{1-r}\right)\right\} .
$$

Now we consider two cases.

Case I. $H>2$.

For $r \in E$, we deduce

$$
\begin{aligned}
\frac{d H}{d r} & =\int_{\theta_{1}+2 \varepsilon}^{\theta_{2}-2 \varepsilon} \log +\frac{\left\|\vec{A}\left(r e^{i \theta}\right)\right\|\|\vec{a}\|}{\left|F\left(r e^{i \theta}, a\right)\right|} d \theta \\
& >2 \pi\left(\frac{1}{1-r}\right)^{\rho+1 / \omega+3+2 \varepsilon} \log ^{2}\left(\frac{1}{1-r}\right) \\
& \geq 2 \pi H \kappa \log ^{2} H \frac{1}{1-r},
\end{aligned}
$$

where $\kappa$ is a positive constant.

From (2.14) it follows that

$$
\int_{E} \frac{d r}{1-r} \leq \int_{2}^{\infty} \frac{d H}{2 \pi H \kappa \log ^{2} H}=O(1) .
$$

Case II. $H \leq 2$.

Since $H$ increases as $r \rightarrow 1-$, we obtain

$$
\lim _{r \rightarrow 1-} H=\int_{2^{-1}}^{1} \int_{\theta_{1}+2 \varepsilon}^{\theta_{2}-2 \varepsilon} \log ^{+} \frac{\left\|\vec{A}\left(r e^{i \theta}\right)\right\|\|\vec{a}\|}{\left|F\left(r e^{i \theta}, a\right)\right|} d \theta d r \leq 2 .
$$


For $r \in E$, we deduce

$$
\frac{1}{1-r}<\left(\frac{1}{1-r}\right)^{\rho+1 / \omega+3+2 \varepsilon} \log ^{2}\left(\frac{1}{1-r}\right)<\frac{1}{2 \pi} \int_{\theta_{1}+2 \varepsilon}^{\theta_{2}-2 \varepsilon} \log ^{+} \frac{\left\|\vec{A}\left(r e^{i \theta}\right)\right\|\|\vec{a}\|}{\left|F\left(r e^{i \theta}, a\right)\right|} d \theta .
$$

Therefore, we have

$$
\begin{aligned}
\int_{E} \frac{d r}{1-r} \leq & \int_{E} \frac{1}{2 \pi} \int_{\theta_{1}+2 \varepsilon}^{\theta_{2}-2 \varepsilon} \log +\frac{\left\|\vec{A}\left(r e^{i \theta}\right)\right\|\|\vec{a}\|}{\left|F\left(r e^{i \theta}, a\right)\right|} d \theta d r \\
\leq & \int_{E \cap\left[0,2^{-1}\right]} \frac{1}{2 \pi} \int_{\theta_{1}+2 \varepsilon}^{\theta_{2}-2 \varepsilon} \log ^{+} \frac{\left\|\vec{A}\left(r e^{i \theta}\right)\right\|\|\vec{a}\|}{\left|F\left(r e^{i \theta}, a\right)\right|} d \theta d r \\
& +\int_{E \cap\left[2^{-1}, 1\right)} \frac{1}{2 \pi} \int_{\theta_{1}+2 \varepsilon}^{\theta_{2}-2 \varepsilon} \log ^{+} \frac{\left\|\vec{A}\left(r e^{i \theta}\right)\right\|\|\vec{a}\|}{\left|F\left(r e^{i \theta}, a\right)\right|} d \theta d r \\
\leq & \int_{0}^{2^{-1}} \frac{1}{2 \pi} \int_{\theta_{1}+2 \varepsilon}^{\theta_{2}-2 \varepsilon} \log ^{+} \frac{\left\|\vec{A}\left(r e^{i \theta}\right)\right\|\|\vec{a}\|}{\left|F\left(r e^{i \theta}, a\right)\right|} d \theta d r+\frac{2}{\pi}<\infty .
\end{aligned}
$$

Thus the proof of Lemma 2.4 is completed.

In order to establish the small arc theorem for the algebroid functions, we have the following lemma first. Indeed, the proof has been given in [12]. For completeness, we give the proof here again.

LEMMA 2.5. Let $f(\xi)$ be the $v$-valued algebroid function in $\Delta$ determined by (1.1). Then, for any $z=r e^{i \theta}, 0<r<R<1$, we have

$$
\log ^{+} \frac{\|\vec{A}(z)\|\|\vec{a}\|}{|F(z, a)|} \leq \log ^{+}(v+1)^{1 / 2}+\frac{R+r}{R-r} m(R, \vec{a}, \vec{A})+\sum_{t=1}^{M} \log \left|\frac{2 R}{z-b_{t}}\right|,
$$

where $b_{1}, b_{2}, \ldots, b_{M}$ are the roots of the equation $f(\xi)=a$ in $|\xi|<R$.

PROOF. We will prove that (2.15) holds for every point $z$. For any $z=r e^{i \theta}, 0<r<$ $R<1$, there exists an integer $0 \leq k=k_{z} \leq v$, such that

$$
\max _{0 \leq l \leq v}\left|A_{l}(z)\right|=\left|A_{k}(z)\right| \text {. }
$$

Then

$$
\begin{aligned}
\log ^{+} \frac{\|\vec{A}(z)\|\|\vec{a}\|}{|F(z, a)|} & \leq \log ^{+} \frac{(v+1)^{1 / 2}\left|A_{k}(z)\right|\|\vec{a}\|}{|F(z, a)|} \\
& \leq \log ^{+}(v+1)^{1 / 2}+\log ^{+} \frac{\left|A_{k}(z)\right|\|\vec{a}\|}{|F(z, a)|} \\
& =\log ^{+}(v+1)^{1 / 2}+\log ^{+}\left|\frac{A_{k}(z)}{F(z, a)}\|\vec{a}\|\right| .
\end{aligned}
$$

Notice that both $A_{k}(\xi)$ and $F(\xi, a)$ are entire functions, $\|\vec{a}\|$ is a constant number, then

$$
\frac{A_{k}(\xi)\|\vec{a}\|}{F(\xi, a)}
$$


is a meromorphic function. Now we apply the Possion-Jensen formula to the meromorphic function $A_{k}(\xi)\|\vec{a}\| / F(\xi, a)$ and have a estimation:

$$
\begin{aligned}
\log ^{+}\left|\frac{A_{k}(z)\|\vec{a}\|}{F(z, a)}\right| \leq & \frac{1}{2 \pi} \int_{0}^{2 \pi} \log ^{+}\left|\frac{A_{k}\left(R e^{i \phi}\right)\|\vec{a}\|}{F\left(R e^{i \phi}, a\right)}\right| \frac{R^{2}-r^{2}}{R^{2}-2 R r \cos (\theta-\phi)+r^{2}} d \phi \\
& +\sum_{t=1}^{M} \log \left|\frac{R^{2}-\bar{b}_{t} z}{R\left(z-b_{t}\right)}\right| .
\end{aligned}
$$

From the inequality $\frac{R^{2}-r^{2}}{R^{2}-2 R r \cos (\theta-\phi)+r^{2}} \leq \frac{R+r}{R-r}$, we derive

$$
\log ^{+}\left|\frac{A_{k}(z)\|\vec{a}\|}{F(z, a)}\right| \leq \frac{1}{2 \pi} \frac{R+r}{R-r} \int_{0}^{2 \pi} \log ^{+} \frac{\left|A_{k}\left(R e^{i \phi}\right)\right|\|\vec{a}\|}{\left|F\left(\operatorname{Re}^{i \phi}, a\right)\right|} d \phi+\sum_{t=1}^{M} \log \left|\frac{2 R}{z-b_{t}}\right| .
$$

From $\left|A_{k}\left(R e^{i \phi}\right)\right| \leq\left\|\vec{A}\left(R e^{i \phi}\right)\right\|$, we have

$$
\log ^{+}\left|\frac{A_{k}(z)\|\vec{a}\|}{F(z, a)}\right| \leq \frac{R+r}{R-r} m(R, \vec{a}, \vec{A})+\sum_{t=1}^{M} \log \left|\frac{2 R}{z-b_{t}}\right| .
$$

This completes the proof.

Using the method of [2], we can establish Lemma 2.6, which is called the small arc theorem for algebroid functions.

LEMMA 2.6. Let $I(r) \subset[0,2 \pi)$ be a measurable set of $\theta$ with its measure $|I(r)|$. Then for $0<r<R<1$, we have

$$
\frac{1}{2 \pi} \int_{I(r)} \log ^{+} \frac{\left\|\vec{A}\left(r e^{i \theta}\right)\right\|\|\vec{a}\|}{\left|F\left(r e^{i \theta}, a\right)\right|} d \theta \leq \frac{K R}{R-r} T(R, f)|I(r)|\left(1+\log ^{+} \frac{1}{|I(r)|}\right),
$$

where $K$ is a positive constant number and is independent of $r, \theta$.

PROOF. Let $\left\{b_{l}=\left|b_{l}\right| e^{i \beta_{l}}\right\}$ denote the sequence of the roots of $f(z)=a$ in the unit disk. From (2.15), we have

$$
\log ^{+} \frac{\left\|\vec{A}\left(r e^{i \theta}\right)\right\|\|\vec{a}\|}{\left|F\left(r e^{i \theta}, a\right)\right|} \leq \log ^{+}(v+1)^{1 / 2}+\frac{t+r}{t-r} m(t, \vec{a}, \vec{A})+\sum_{\left|b_{l}\right| \leq t} \log \left|\frac{2 t}{r e^{i \theta}-b_{l}}\right|, \quad(r<t)
$$

and note that

$$
\begin{gathered}
\left|r e^{i \theta}-\right| b_{l}\left|e^{i \beta_{l}}\right| \geq r\left|\sin \left(\theta-\beta_{l}\right)\right| \quad\left(\left|\theta-\beta_{l}\right| \leq \pi / 2\right), \\
\left|r e^{i \theta}-\right| b_{l}\left|e^{i \beta_{l}}\right| \geq r \quad\left(\pi / 2<\left|\theta-\beta_{l}\right| \leq \pi\right) .
\end{gathered}
$$

Hence

$$
\begin{aligned}
& \int_{I(r)} \log ^{+} \frac{\left\|\vec{A}\left(r e^{i \theta}\right)\right\|\|\vec{a}\|}{\left|F\left(r e^{i \theta}, a\right)\right|} d \theta \leq|I(r)| \log ^{+}(v+1)^{1 / 2}+|I(r)| \frac{t+r}{t-r} m(t, \vec{a}, \vec{A}) \\
& +\sum_{\left|b_{l}\right| \leq t}\left[\int_{I(r)} \log \left(\frac{2 t}{r}\right) d \theta+\int_{I^{*}\left(r, \beta_{l}\right)} \log \frac{1}{\left|\sin \left(\theta-\beta_{l}\right)\right|} d \theta\right]
\end{aligned}
$$


where $I^{*}\left(r, \beta_{l}\right)$ denotes the portion of $I(r)$ which belongs to the arc $\left[-\pi / 2+\beta_{l}, \beta_{l}+\pi / 2\right]$. Following the inequality (9.4) of pp.338 in [2], we have

$$
\begin{gathered}
\int_{I(r)} \log ^{+} \frac{\left\|\vec{A}\left(r e^{i \theta}\right)\right\|\|\vec{a}\|}{\left|F\left(r e^{i \theta}, a\right)\right|} d \theta \leq|I(r)|\left(\log ^{+}(v+1)^{1 / 2}+\frac{t+r}{t-r} m(t, \vec{a}, \vec{A})\right) \\
+|I(r)| n(t, a, f)\left[\log \left(\frac{2 t}{r}\right)+1+\log ^{+} \frac{\pi}{|I(r)|}\right] \quad(0<r<t) .
\end{gathered}
$$

By the definition of $N(r, a, f)$ and $T(r, f)$,

$$
n(t, a, f) \leq \frac{N(R, a, f)}{\log (R / t)} \leq \frac{\nu T(R, f)}{\log (R / t)} \quad(0<r<t<R<1) .
$$

We choose $t=(r+R) / 2$. Then combining (2.17) with (2.18), we derive

$$
\begin{aligned}
& \int_{I(r)} \log ^{+} \frac{\left\|\vec{A}\left(r e^{i \theta}\right)\right\|\|\vec{a}\|}{\left|F\left(r e^{i \theta}, a\right)\right|} d \theta \leq|I(r)|\left(\log ^{+}(\nu+1)^{1 / 2}+\frac{4 v}{R-r} T(R, f)\right) \\
& +|I(r)| \frac{\nu T(R, f)}{\log (R / t)}\left[\log \left(1+\frac{R}{r}\right)+1+\log ^{+} \frac{\pi}{|I(r)|}\right] .
\end{aligned}
$$

In view of $\log (R / r)>(R-r) / R$, we obtain the result.

Hayman [5] established Lemma 2.7 for a real function defined in $(0,+\infty)$.

LEMMA 2.7 ([5]). Let $T(r)$ be a continuous function of $r$ in $(0,+\infty)$ which increases to $+\infty$ and is of finite lower order $\mu<+\infty$. Then, for two arbitrary positive real number $\tau_{1}$ and $\tau_{2}$ satisfying $\tau_{2}>\tau_{1}>1$, the lower logarithmic density of the set $G=\{r \in$ $\left.(0, \infty) ; T\left(\tau_{1} r\right) \leq \tau_{2} T(r)\right\}$ satisfies

$$
\underline{\log \text { dense }} G=\liminf _{r \rightarrow \infty} \frac{\int_{G \cap[1, r]} \frac{d t}{t}}{\log r} \geq 1-\mu \frac{\log \tau_{1}}{\log \tau_{2}},
$$

where $\mu=\liminf _{r \rightarrow \infty} \log T(r) / \log r$.

Using Lemma 2.7 and by the method of transformation, we can establish Lemma 2.8 .

LEMMA 2.8. Let $T_{1}(r)$ be a continuous function of $r$ in $(0,1)$ which increases to $+\infty$ and is of finite lower order $\mu<+\infty$. Then, for two arbitrary positive real number $\tau_{1}$ and $\tau_{2}$ satisfying $\tau_{2}>\tau_{1}>1$, the lower logarithmic density of the set $G=\left\{r \in(0,1) ; T_{1}(1-\right.$ $\left.\left.\tau_{1}^{-1}+r \tau_{1}^{-1}\right) \leq \tau_{2} T_{1}(r)\right\}$ satisfies

$$
\underline{\log \text { dense }} G=\liminf _{r \rightarrow 1-} \frac{\int_{G \cap[0, r]} \frac{d t}{1-t}}{\log \frac{1}{1-r}} \geq 1-\mu \frac{\log \tau_{1}}{\log \tau_{2}} .
$$

Proof. Set $r=1-t^{-1}$, then $T(t)=T_{1}\left(1-t^{-1}\right)$ is a function defined in $(1, \infty)$. The order and lower order of $T(t)$ equal to the order and lower order of $T_{1}(r)$, i.e.,

$$
\limsup _{t \rightarrow \infty} \frac{\log ^{+} T(t)}{\log t}=\limsup _{t \rightarrow \infty} \frac{\log ^{+} T_{1}\left(1-t^{-1}\right)}{\log t}=\limsup _{r \rightarrow 1-} \frac{\log ^{+} T_{1}(r)}{\log (1-r)^{-1}},
$$




$$
\liminf _{t \rightarrow \infty} \frac{\log ^{+} T(t)}{\log t}=\liminf _{t \rightarrow \infty} \frac{\log ^{+} T_{1}\left(1-t^{-1}\right)}{\log t}=\liminf _{r \rightarrow 1-} \frac{\log ^{+} T_{1}(r)}{\log (1-r)^{-1}} .
$$

Since the lower order of $T_{1}(r)$ is $\mu<+\infty$, the lower order of $T(t)$ is also $\mu<+\infty$. For any $\tau_{2}>\tau_{1}>1$, the set $G$ is mapped onto $\widetilde{G}=\left\{t \in(1, \infty) ; T\left(\tau_{1} t\right) \leq \tau_{2} T(t)\right\}$ under the transformation $t=(1-r)^{-1}$. Now by Lemma 2.7, we have

$$
\liminf _{t \rightarrow \infty} \frac{\int \widetilde{G} \cap[1, t] \frac{d x}{x}}{\log t} \geq 1-\mu \frac{\log \tau_{1}}{\log \tau_{2}}
$$

Under the one to one transformation $r=1-t^{-1}$, the set $\widetilde{G}$ is mapped onto $G$, and $\int_{\widetilde{G} \cap[1, t]} d x / x=\int_{G \cap[0, r]} d x /(1-x)$, then from (2.19) we have

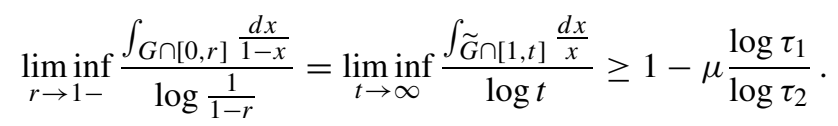

Hence we get the result of the lemma.

LEMMA 2.9. Let $f(z)$ be the $v$-valued algebroid function of finite lower order $\mu$ in $\Delta$ determined by (1.1) and such that for some $a \in \widehat{\boldsymbol{C}}, \delta(a, f)>0$. Suppose that there exist $2 v$ distinct complex values $a_{j} \neq a(j=1,2, \ldots, 2 v)$ such that

$$
\limsup _{r \rightarrow 1-} \frac{\log \left[\sum_{j=1}^{2 v} n\left(r, X, f=a_{j}\right)+n(r, X, f=a)\right]}{\log (1-r)^{-1}} \leq \rho+1<\infty,
$$

where $X=\Delta \backslash \bigcup_{k=1}^{q}\left\{z ; \arg z=\theta_{k},|z|<1\right\}, \rho>0$. Then, for any $\tau_{1}, \tau_{2}$ satisfying $\tau_{2}>$ $\tau_{1}>1$, we have

$$
\limsup _{r \in E_{1} \backslash E, r \rightarrow 1-} \frac{\log ^{+} T(r, f)}{\log (1-r)^{-1}} \leq \rho+3+\max _{1 \leq k \leq q}\left\{\frac{\theta_{k+1}-\theta_{k}}{\pi}\right\},
$$

where $E_{1}=\left\{r ; T\left(1-\tau_{1}^{-1}+\tau_{1}^{-1} r, f\right) \leq \tau_{2} T(r, f)\right\} \subset(0,1)$ and $E \subset(0,1)$ is satisfying that $\int_{E} d r /(1-r)<\infty$.

Proof. From Lemma 2.4 and the condition of Lemma 2.9, it follows that

$$
\begin{aligned}
& \sum_{k=1}^{q} \frac{1}{2 \pi} \int_{\theta_{k}+2 \varepsilon}^{\theta_{k+1}-2 \varepsilon} \log ^{+} \frac{\left\|\vec{A}\left(r e^{i \theta}\right)\right\|\|\vec{a}\|}{\left|F\left(r e^{i \theta}, a\right)\right|} d \theta \\
& \quad=O\left(\sum_{k=1}^{q}\left(\frac{1}{1-r}\right)^{\rho+1 / \omega_{k}+3+2 \varepsilon} \log ^{2} \frac{1}{1-r}\right)
\end{aligned}
$$


where $r \in(0,1) \backslash E, \int_{E} d r /(1-r)<\infty, \omega_{k}=\pi /\left(\theta_{k+1}-\theta_{k}-2 \varepsilon\right)$.

Setting $R=1-\tau_{1}^{-1}+\tau_{1}^{-1} r$ in Lemma 2.6 and noticing that $r \in E_{1}$, we obtain

$$
\begin{aligned}
\sum_{k=1}^{q} & \frac{1}{2 \pi}\left(\int_{\theta_{k}}^{\theta_{k}+2 \varepsilon}+\int_{\theta_{k+1}-2 \varepsilon}^{\theta_{k+1}}\right) \log ^{+} \frac{\left\|\vec{A}\left(r e^{i \theta}\right)\right\|\|\vec{a}\|}{\left|F\left(r e^{i \theta}, a\right)\right|} d \theta \\
& \leq \frac{K}{1-r} T\left(1-\tau_{1}^{-1}+\tau_{1}^{-1} r, f\right) 4 q \varepsilon\left(1+\log ^{+} \frac{1}{2 \varepsilon}\right) \\
& \leq \frac{K}{1-r} \tau_{2} T(r, f) 4 q \varepsilon\left(1+\log ^{+} \frac{1}{2 \varepsilon}\right) .
\end{aligned}
$$

Taking $\varepsilon=\varepsilon(r)=(1-r)^{2}$ in (2.22) yields

$$
\begin{aligned}
\sum_{k=1}^{q} & \frac{1}{2 \pi}\left(\int_{\theta_{k}}^{\theta_{k}+2 \varepsilon}+\int_{\theta_{k+1}-2 \varepsilon}^{\theta_{k+1}}\right) \log ^{+} \frac{\left\|\vec{A}\left(r e^{i \theta}\right)\right\|\|\vec{a}\|}{\left|F\left(r e^{i \theta}, a\right)\right|} d \theta \\
& \leq 4 q K(1-r)\left(1+\log ^{+} \frac{1}{2(1-r)^{2}}\right) \tau_{2} T(r, f) \\
& <\frac{\nu \delta(a, f)}{4} T(r, f), \quad r \rightarrow 1-.
\end{aligned}
$$

Setting in (2.21) $\varepsilon=\varepsilon(r)=(1-r)^{2}$ and adding together (2.21) and (2.23), we derive

$$
m(r, \vec{a}, \vec{A}) \leq \frac{\nu \delta(a, f)}{4} T(r, f)+O\left(\sum_{k=1}^{q}\left(\frac{1}{1-r}\right)^{\rho+1 / \omega_{k}+3+2 \varepsilon} \log ^{2} \frac{1}{1-r}\right), \quad r \in E_{1} \backslash E .
$$

By the definition of $\delta(a, f)$, we have

$$
m(r, \vec{a}, \vec{A})>\frac{\nu \delta(a, f)}{2} T(r, f), \quad r \rightarrow 1-.
$$

So that

$$
\frac{\nu \delta(a, f)}{4} T(r, f) \leq O\left(\sum_{k=1}^{q}\left(\frac{1}{1-r}\right)^{\rho+1 / \omega_{k}+3+2 \varepsilon} \log ^{2} \frac{1}{1-r}\right), \quad r \in E_{1} \backslash E, r \rightarrow 1-.
$$

This leads to

$$
\limsup _{r \in E_{1} \backslash E, r \rightarrow 1-} \frac{\log ^{+} T(r, f)}{\log (1-r)^{-1}} \leq \rho+3+\max _{1 \leq k \leq q}\left\{\frac{\theta_{k+1}-\theta_{k}}{\pi}\right\} .
$$

The proof is completed.

\section{Proof of Theorem 1.1. Set}

$$
K:=\rho+2+\max _{1 \leq k \leq q}\left\{\frac{\theta_{k+1}-\theta_{k}}{\pi}\right\} .
$$

By Lemma 2.9, we have

$$
\limsup _{r \in E_{1} \backslash E, r \rightarrow 1-} \frac{\log ^{+} T(r, f)}{\log (1-r)^{-1}} \leq K,
$$


where $\underline{\log \text { dens }} E_{1} \geq 1-\mu \frac{\log \tau_{1}}{\log \tau_{2}}, \int_{E} \frac{d r}{1-r}<\infty, \mu$ is the lower order of $f$. Obviously, $\mu \leq K$. Choose $\tau_{2}$ large enough such that

$$
\log \tau_{2}>3 K \log \tau_{1} \geq 3 \mu \log \tau_{1} .
$$

So that

$$
\begin{aligned}
\liminf _{r \rightarrow 1-} \frac{\int_{\left(E_{1} \backslash E\right) \cap[0, r] \frac{d t}{1-t}}}{\log \frac{1}{1-r}} & \geq \liminf _{r \rightarrow 1-} \frac{\int_{E_{1} \cap[0, r]} \frac{d t}{1-t}}{\log \frac{1}{1-r}}-\limsup _{r \rightarrow 1-} \frac{\int_{E \cap[0, r]} \frac{d t}{1-t}}{\log \frac{1}{1-r}} \\
& =1-\mu \frac{\log \tau_{1}}{\log \tau_{2}}-0>\frac{2}{3} .
\end{aligned}
$$

This leads to $\lim \sup _{r \rightarrow 1-, r \in E_{1} \backslash E}\{r\}=1$. Thus $r \in E_{1} \backslash E, r \rightarrow 1-$ can hold.

Set $F=E_{1} \backslash E$. Now we claim that for $r \rightarrow 1-, r \notin F$, there exists a number $r^{\prime}$ such that $r^{\prime} \in F$ and $r^{\prime} \in\left[r, 1-(1-r)^{d}\right]$, where

$$
d=1+\frac{3 K \log \tau_{1}}{\log \tau_{2}}<2 .
$$

In fact, otherwise, there exists a sequence $\left\{r_{n}\right\}$ such that $r_{n} \rightarrow 1$ as $n \rightarrow \infty$ and $F \cap\left[r_{n}, 1-\right.$ $\left.\left(1-r_{n}\right)^{d}\right]=\emptyset$ for each $n$. So that

$$
F \subset(0,1) \backslash \bigcup_{n=1}^{\infty}\left[r_{n}, 1-\left(1-r_{n}\right)^{d}\right] .
$$

Assume $r_{n}>1-\left(1-r_{n-1}\right)^{d}$ (otherwise we can consider a subsequence), then

$$
\begin{aligned}
\int_{F \cap\left[0,1-\left(1-r_{n}\right)^{d}\right]} \frac{d t}{1-t} & \leq \int_{\left[0,1-\left(1-r_{n}\right)^{d}\right] \backslash \bigcup_{j=1}^{n}\left[r_{j}, 1-\left(1-r_{j}\right)^{d}\right]} \frac{d t}{1-t} \\
& =\sum_{j=2}^{n} \int_{1-\left(1-r_{j-1}\right)^{d}}^{r_{j}} \frac{d t}{1-t} \\
& =\log \frac{1}{1-r_{n}}-(d-1) \sum_{j=2}^{n-1} \log \frac{1}{1-r_{j}}-d \log \frac{1}{1-r_{1}} .
\end{aligned}
$$

On the other hand, by (3.3), we have for $r_{n} \rightarrow 1-$,

$$
\frac{1}{d \log \frac{1}{1-r_{n}}} \int_{F \cap\left[0,1-\left(1-r_{n}\right)^{d}\right]} \frac{d t}{1-t} \geq 1-K \frac{\log \tau_{1}}{\log \tau_{2}}=1-\frac{d-1}{3} .
$$

Combining (3.4) with (3.5), we have

$$
0<\sum_{j=2}^{n-1} \frac{\log \left(1-r_{j}\right)^{-1}}{\log \left(1-r_{n}\right)^{-1}} \leq \frac{d}{3}-1<\frac{2}{3}-1 .
$$

This is impossible.

Since $r^{\prime} \in F$, we have

$$
T\left(r^{\prime}, f\right)<\left(\frac{1}{1-r^{\prime}}\right)^{K+\varepsilon}
$$


by Lemma 2.9. Consequently,

$$
T(r, f) \leq T\left(r^{\prime}, f\right) \leq\left(\frac{1}{1-r^{\prime}}\right)^{K+\varepsilon} \leq\left(\frac{1}{1-r}\right)^{\left(1+\frac{3 K \log \tau_{1}}{\log \tau_{2}}\right)(K+\varepsilon)}
$$

Taking limitation,

$$
\limsup _{r \rightarrow 1-} \frac{\log ^{+} T(r, f)}{\log (1-r)^{-1}} \leq\left(1+\frac{3 K \log \tau_{1}}{\log \tau_{2}}\right)(K+\varepsilon) .
$$

Letting $\varepsilon \rightarrow 0, \tau_{2} \rightarrow \infty$,

$$
\limsup _{r \rightarrow 1-} \frac{\log ^{+} T(r, f)}{\log (1-r)^{-1}} \leq K=\rho+2+\max _{1 \leq k \leq q}\left\{\frac{\theta_{k+1}-\theta_{k}}{\pi}\right\} .
$$

We complete the proof of the theorem.

Acknowledgments. The authors would like to express their many thanks to the referee for the valuable comments and suggestions in improving this paper.

The first author is supported by the grant (No. 11231009, 11371363, 11326086) of NSF of China.

The second author is supported by the grant (No. 11171170) of NSF of China and the research fund (No. 20100002110012) for the Doctoral Program of Higher Education, Ministry of Education of China.

\section{REFERENCES}

[ 1 ] J. M. ChAng, Radially distributed values of meromorphic functions, J. Math. Anal. Appl. 319 (2006), 34-44.

[2] A. EDREI AND W. H. J. FUCHS, Bounds for the number of deficient value of certain classes of meromorphic functions, Proc. London Math. Soc. 12 (1962), 315-344.

[ 3 ] A. EDREI, Meromorphic functions with three radially distributed values, Trans. Amer. Math. Soc. 78 (1955), 276-293.

[ 4 ] W. K. Hayman, Meromorphic functions, Oxford Math. Monogr., Clarendon Press, Oxford, 1964.

[ 5 ] W. K. Hayman, Angular value distribution of power series with gaps, Proc. London Math. Soc. 24 (1972), 590-624.

[6] Y. Z. He AND X. Z. XIAO, Algebroid functions and ordinary differential equations (in Chinese), Science Press of China, Beijing, 1988.

[ 7 ] D. C. Sun And J. R. Yu, On the distribution of values of random Dirichlet series. II, Chinese Ann. Math. Ser. B 11 (1990), 33-44.

[ 8 ] N. TodA, Sur les directions de Julia et de Borel des fonctions algébroıdes, Nagoya Math. J. 34 (1969), 1-23.

[9] M. TsuJi, Potential theory in modern function theory, Maruzen Co. LTD., Tokyo, 1959.

[10] S. J. WU, Angular distribution and growth of meromorphic functions, Sci. China Ser. A 36 (1993), 791-802.

[11] N. WU AND Z. X. XUAN, Common Borel radii of an algebroid function and its derivative, Results Math. 62 (2012), no. 1-2, 89-101.

[12] N. Wu AND J. H. Zheng, On the growth of an algebroid function with radially distributed values, Ann. Pol. Math. to appear.

[13] L. YANG, Value distribution and new research, Springer-Verlag, Berlin, 1993.

[14] L. YANG AND C. C. YANG, Angular distribution of values of $f f^{\prime}$, Sci. China Ser. A 37 (1994), $284-294$.

[15] L. Z. YANG, Sums of deficiencies of algebroid functions, Bull. Austral. Math. Soc. 42 (1990), 191-200.

[16] Q. D. ZHANG, Distribution of Borel radii of meromorphic functions in the unit disc (in Chinese), Acta Math. Sinica (Chin. Ser.) 42 (1999), no. 2, 351-358.

[17] J. H. ZHENG, Value distribution of meromorphic functions, Springer-Verlag, Berlin, 2010. 
[18] J. H. ZHENG, On value distribution of meromorphic functions with respect to arguments I, Complex Var. Elliptic Equ. 56 (2011), 271-298.

[19] J. H. ZHENG, On transcendental meromorphic functions with radially distributed values, Sci. China Ser. A 47 (2004), 401-416.

DEPARTMENT OF MATHEMATICS SCHOOL OF SCIENCE

CHINA UNIVERSITY OF MINING AND TECHNOLOGY (BEIJING)

BEIJING, 100083

People's Republic of ChinA

E-mail address: wunan2007@163.com
Department of Mathematical Sciences

TSINGHUA UNIVERSITY

BEIJING, 100084

PEOPLE's REPUBLIC OF CHINA

E-mail address: jzheng@math.tsinghua.edu.cn 